\title{
Map the apps: a rapid review of digital approaches to support the engagement of older adults in strength and balance exercises
}

Dol:

10.1186/s12877-020-01880-6

\section{Document Version}

Final published version

Link to publication record in Manchester Research Explorer

Citation for published version (APA):

McGarrigle PhD, L., Boulton, E., \& Todd, C. (2020). Map the apps: a rapid review of digital approaches to support the engagement of older adults in strength and balance exercises. BMC Geriatrics, 20(483).

https://doi.org/10.1186/s12877-020-01880-6

\section{Published in:}

BMC Geriatrics

\section{Citing this paper}

Please note that where the full-text provided on Manchester Research Explorer is the Author Accepted Manuscript or Proof version this may differ from the final Published version. If citing, it is advised that you check and use the publisher's definitive version.

\section{General rights}

Copyright and moral rights for the publications made accessible in the Research Explorer are retained by the authors and/or other copyright owners and it is a condition of accessing publications that users recognise and abide by the legal requirements associated with these rights.

\section{Takedown policy}

If you believe that this document breaches copyright please refer to the University of Manchester's Takedown Procedures [http://man.ac.uk/04Y6Bo] or contact uml.scholarlycommunications@manchester.ac.uk providing relevant details, so we can investigate your claim.

\section{OPEN ACCESS}




\title{
Map the apps: a rapid review of digital approaches to support the engagement of older adults in strength and balance exercises
}

\author{
Lisa McGarrigle 1,2* $^{*}$, Elisabeth Boulton ${ }^{1,2,3}$ and Chris Todd ${ }^{1,2,3,4,5}$
}

\begin{abstract}
Background: Exercise interventions, particularly those targeting strength and balance, are effective in preventing falls in older people. Activity levels are generally below recommended levels and reduce with age. There is concern that exercise levels may be further reduced in the context of the COVID-19 pandemic. Digital approaches may offer a means for older people to engage in strength and balance exercises independently in their own homes. The objective of this review was to identify and evaluate existing apps and websites to support independent engagement in strength and balance exercises by older people.

Methods: We conducted a rapid review of apps and websites, following PRISMA guidelines. We searched for available apps in the Android and iOS app stores, and performed a database search (MEDLINE and EMBASE) for apps in development. We searched for websites using the Google search engine. Apps and websites were evaluated in terms of existing evidence for effectiveness, use of behaviour change techniques (BCTs), and quality.

Results: We evaluated 13 apps and 24 websites on the basis of our selection criteria. Considering the evidence-base, quality and BCT scores, four apps and six websites are recommended for use by older people who wish to engage in exercise independently in their own homes. No apps or websites have been to RCT evaluation at the time of review.

Conclusions: Apps and websites have the potential to provide a convenient, cost-effective, and accessible means for many older adults to engage in strength and balance training and reduce falls risk.
\end{abstract}

Keywords: Apps, Websites, Strength, Balance, Exercise, Behaviour change techniques, Falls prevention, COVID-19

\section{Background}

Worldwide, falls are the second leading cause of accidental death, with those aged over 65 experiencing the largest proportion of fatal falls [1]. Injury resulting from falls is associated with reduced physical functioning, loss of independence, and fear of future falls, which can in

\footnotetext{
* Correspondence: lisa.mcgarrigle@manchester.ac.uk

'School of Health Sciences, Faculty of Biology, Medicine and Health, The University of Manchester, Jean McFarlane Building, University Place, Oxford Road, Manchester M13 9PL, UK

${ }^{2}$ Manchester Academic Health Science Centre, Manchester M13 9NQ, UK Full list of author information is available at the end of the article
}

turn lead to reductions in physical activity and social engagement $[2,3]$. A major causal factor in falls amongst older people is reduced muscle mass and strength resulting from metabolic changes and low levels of physical activity [4]. Exercise interventions, particularly those targeting strength and balance, can be effective in preventing falls in older people [5]. Recent UK Chief Medical Officers' guidelines state that all adults aged 65 and over should aim to perform muscle-strengthening and balance exercises at least 2 days a week [6]. In the UK, rates of engagement in strength and balance activities are

(c) The Author(s). 2020 Open Access This article is licensed under a Creative Commons Attribution 4.0 International License, which permits use, sharing, adaptation, distribution and reproduction in any medium or format, as long as you give appropriate credit to the original author(s) and the source, provide a link to the Creative Commons licence, and indicate if changes were made. The images or other third party material in this article are included in the article's Creative Commons licence, unless indicated otherwise in a credit line to the material. If material is not included in the article's Creative Commons licence and your intended use is not permitted by statutory regulation or exceeds the permitted use, you will need to obtain permission directly from the copyright holder. To view a copy of this licence, visit http://creativecommons.org/licenses/by/4.0/ The Creative Commons Public Domain Dedication waiver (http://creativecommons.org/publicdomain/zero/1.0/) applies to the data made available in this article, unless otherwise stated in a credit line to the data. 
generally low, with only one in four women and one in three men over the age of 19 meeting the recommended guidelines, and the number meeting the recommendations decreases with age [7].

During the worldwide COVID-19 pandemic of 2020 there is concern that stay at home measures to reduce transmission of coronavirus will have adverse effects on older adults' levels of activity with resultant deconditioning and increased falls risk. There is evidence from systematic reviews to suggest that exercise interventions delivered via apps and websites are successful in increasing physical activity levels in older people $[8,9]$, but similar evidence in relation to falls outcomes is lacking. There is also evidence that strength and balance training delivered via exergaming can improve strength and balance and reduce falls risk [10, 11], however, these kinds of interventions might not be immediately available to the general public. Given the risk the current pandemic restrictions pose to older people in relation to reduced activity, easily accessible and publicly available apps and websites that demonstrate strength and balance exercises via videos or images could act as potential substitutes in the absence of face-to-face exercise programmes. However, evidence for their effectiveness in falls prevention is unclear.

The objective of this review is to identify publicly available digital resources, in the form of apps and websites that can support older adults in performing strength and balance exercises independently, and to evaluate their quality, and evidence for effectiveness in improving strength and balance and/or preventing falls.

\section{Methods}

We conducted a rapid review of apps and websites that support older adults in performing strength and balance exercises. The Prevention of Falls Network Europe (ProFaNE) [12] developed a taxonomy to classify different types of exercises. Strength exercises involve training the muscular system using free weights, body weight, or resistance, and may often involve an element of balance. Strengthening of the lower body is particularly important in falls prevention and examples of strength exercises may include heel and toe raises, knee bends, and sit to stand [13]. Exercises designed to improve balance involve the transfer of bodyweight from one part of the body to another and can include a wide variety of dynamic movements, such as heel and toe raises, squats, calf raises, and standing on one leg or tandem standing [12]. Although there is some evidence that " $3 D$ " exercises that involve constant movement, such as Tai Chi are also effective in falls prevention [5], for the purposes of this review we focused only on strength and balance exercises as outlined above. The review followed PRIS MA guidelines. Rapid reviews allow for the synthesis of evidence relating to a specific query in a timely fashion $[14,15]$. A protocol was registered on PROSPERO (CRD42020178582).

\section{Search strategy and selection criteria}

The rapid review contained two search components: app search and website search. The app search was conducted between November 2019 and February 2020, and was rerun in May 2020 to identify any additional apps that had been recently developed. The website search was conducted in November 2019 and was re-run in May 2020 to ensure findings were up to date. One author (LM) conducted the searches and results were discussed with the other authors $(\mathrm{EB}, \mathrm{CT})$ to agree inclusion/exclusion.

\section{App search}

The first component involved: (a) a search of Android and iOS app stores for apps that met our inclusion criteria; (b) a database search for studies or protocols involving strength and balance exercise apps that are currently in development, but not yet publicly available. The app search was restricted to United Kingdom (UK) app stores. We did not exclude studies found during the database search on the basis of design (randomised controlled trials (RCTs), non-randomised studies or observational studies) in order to gain as full a view of the literature as possible. As this was a rapid review, apps and studies were restricted to the English language to ensure timely review and evidence evaluation.

The search was conducted using the following search strategy:

(a) Seven keyword searches were conducted in the Google Play android app store and the Apple App store. Health and Fitness app categories within each app store were searched using the following seven keywords/terms: falls prevention; balance exercise; balance training; strength exercise; strength training; strength and balance exercise; and strength and balance training. All terms were then combined with 'older adults', 'elderly' and 'seniors', giving a total of 28 searches in each app store.

(b) Database searches were conducted in MEDLINE (Ovid) and EMBASE (Ovid) for studies and protocols involving apps that met our inclusion criteria. Search terms are provided in Additional file 1.

\section{Website search}

The second component involved an internet search to identify websites that contained demonstrations (videos/ images) of falls prevention exercises. The searches were conducted in the UK using the Google search engine. Keyword searches were performed using the following 
seven keywords/terms: fall prevention exercises; exercises to reduce falls; exercises to improve balance; balance training; strength training; strength and balance exercise infographics; and strength and balance exercise demonstration. These terms were searched in isolation and then in combination with 'older adults', 'elderly' and 'seniors', resulting in 28 searches. As research shows that internet users tend to explore only the first few hits from search engines, the first 20 results identified from each search were examined [16-18]. As such, results may not provide a fully comprehensive list of all available webbased falls prevention exercise demonstrations.

\section{Inclusion criteria}

Apps and websites were included in the review if they:

- visually demonstrated strength and/or balance exercises (e.g. using videos or images); this was to ensure that the correct way to conduct the exercise was as clear as possible;

- were publicly available, or were in development with the intention of being made publicly available;

- could be used by older people independently, without supervision by a healthcare professional. As such, we will include apps and websites that target community-dwelling older people.

- apps were appropriate for use with older populations (i.e. were designed to be used by older people aged $\geq 50$ years or had demonstrated use in older populations); the exercises demonstrated via websites were directly targeted at older people in relation to falls prevention;

\section{Exclusion criteria}

We excluded apps and websites if they:

- provided only written descriptions of exercises;

- focused on improving specific disease conditions and falls prevention was a secondary outcome for the app intervention;

- targeted populations other than communitydwelling older people (e.g. patients in acute care facilities or care homes).

- focused on interventions that did not include strength and balance exercises (e.g. cognitive interventions for balance), or focused on general fitness;

- required specialist exercise equipment to work;

- for the website search, we did not consider material solely available on video sharing platforms (e.g. YouTube) or social media platforms (e.g. Facebook; Pinterest).

\section{Evidence evaluation}

The quality of included apps and websites was evaluated as follows:

1. We determined the extent to which the exercises delivered via the app/website were based on evidence-based strength and balance programmes such as the Falls Management Exercise Programme (FaME) [19] or the Otago Exercise Programme (Otago) [20].

2. We determined whether the implementation of the exercise programme was theory-driven, e.g. drew on behaviour change techniques (BCTs) in order to change exercise-related behaviours. Two independent raters assessed this using the taxonomy of BCTs developed by Michie [21]. The taxonomy contains a list of 93 BCTs for which a score of "0" (absent) or " 1 " (present) is applied. Each app was awarded a total score ranging from 0 to 93 , with higher scores indicating greater use of BCTs.

In addition, the quality of apps was assessed by at least two independent raters using a standardised tool for health apps, the Mobile Application Rating Scale (MARS) [22]. The scale contains 23 items assessing engagement, functionality, aesthetics, information quality, and subjective quality, and has demonstrated excellent internal consistency and inter-rater reliability. We also visited the app/developer websites and contacted the developers directly for information on any studies or evaluations that had been conducted in relation to the app. Where studies had been published, we planned to determine study quality using the evidence pyramid as a guide. As we did not anticipate finding any systematic reviews or evidence syntheses relating to the included apps, we considered RCTs as the highest quality evidence. Cohort studies and casecontrolled studies were considered medium quality evidence. Non-randomised studies and studies with no control group were considered low quality evidence. Where possible, we planned to assess RCTs for bias using Cochrane's Risk of Bias tool [23] and non-randomised studies for bias using the ROBINS-I [24]. We did not anticipate that effectiveness studies would have been conducted in relation to websites, so we merely checked the websites for any indications that evaluations had been conducted.

The quality of websites was assessed by two independent raters using two of the three criteria (credibility and senior friendliness) evaluated in Whitehead et al.'s [18] review of falls prevention websites. We did not grade based on the criterion 'coverage of falls information'. This criterion was intended to grade the types of falls prevention information on websites, with information on strength and balance exercises considered Grade A 
information. As all websites were selected on the basis of providing information on strength and balance exercises, this criterion was excluded. Credibility and senior friendliness were assessed as follows:

1. Credibility: Assessed using the Health on the Net Code of Conduct for Medical and Health Websites (HONCode) [25], which consists of eight statements relating to factors such as authority and attribution of information, privacy, and transparency. A score of ' 1 ' was awarded if the statement was satisfied, and ' 0 ' if not. The first criterion, relating to medical advice, was not applicable to any of the websites, and so this item was not used. The score range for credibility was therefore $0-7$.

2. Senior friendliness: Rated using a checklist developed by the National Institute on Aging. Forty requirements are listed across five categories: organising information (8 items); writing online text (11 items); designing readable online text (9 items); making information easy to find (9 items); and including other media ( 3 items). If the requirement was satisfied, a score of "1" was awarded, and a score of "0" if not, giving a score range of 0-40.

Scores for each category were summed to produce a total quality score ranging from 0 to 47 . Percentage scores were calculated, and websites were categorised as poor, fair, good and excellent.

\section{Data analysis}

Inter-rater reliability between two independent raters was assessed for BCT, credibility, and senior friendliness ratings using Krippendorff's Alpha (Kalpha) [26], and all interpretations were based on mean rater scores. MARS ratings were conducted by multiple pairs of raters $(n=$ 6), with only one rater remaining constant for all 13 apps. As such, there was substantial missing data across this small sample and inter-rater reliability analysis was considered inappropriate [27]. Differences in MARS scores, and the mean and standard deviation of the differences, were calculated between raters. Frequencies, means and standard deviations were calculated for the BCTs included in each app and website. Total scores on credibility and senior friendliness were converted into percentages and an overall website quality score was calculated. To ensure both categories were equally represented in the overall score, percentages for each individual category were calculated first, before a total average percentage was calculated. Scores less than 50\% were interpreted as poor, scores between 50 and $62 \%$ as fair, scores between 63 and $75 \%$ as good, and scores greater than $75 \%$ were considered excellent [18]. Where possible, we planned to perform a process evaluation on studies found to have an effective intervention. We planned to summarise and tabulate five key elements outlined by the MRC [28] to identify common pathways. These included: description of the intervention; causal assumptions; implementation; mechanism of impact; and outcomes. Where there was sufficient homogeneity across studies evaluating the apps, we planned to conduct meta-analyses and derive forest plots to compare mean differences and 95\% confidence intervals in rate of falls and number of fallers, as well as mean differences (95\% CIs) across measures of strength, balance, cognitive and psychological factors associated with falling, between intervention and control groups.

\section{Results}

Flow diagrams of identification and retention of apps and websites are presented in Figs. 1 and 2. Overall, 28 apps were considered for inclusion. Reasons for exclusion following the app screening process are listed in Additional file 1. We identified 25 apps from the searches in Google Play and the Apple App Store. Of these, 12 fulfilled inclusion criteria (LifeCurve; StopFalls; Otago Exercise Programme; Spiro100; Nymbl Balance Training; Moves4Me; Stannah Balance; Wysefit; Keep On Keep Up; Exercise Plan for Seniors; Hearty Seniors; and Senior Beginner Workout). We identified three further apps from our database search for apps in development, one intervention study (ActiveLifestyle) [29] and two study protocols (StandingTall and eLiFE) [30,31] involving app-based interventions. Out of these 15 apps, 11 were available for download in UK app stores. We contacted developers to gain access to the remaining four apps, and were granted access to two of them (StandingTall; Nymbl Balance Training). We were unable to gain access to the ActiveLifestyle app and the eLiFE app is not planned to be made publicly available following feasibility RCT testing [32]. Thus, these two apps were excluded from the review. A total of 13 apps were evaluated.

We identified 24 relevant websites for inclusion in the review. As there were no available published studies evaluating the effectiveness of the included apps or websites, we were unable to perform process evaluations, risk of bias assessments, or meta-analyses. Full narrative summaries of all apps and websites detailing the aim, target population, description, platform, and evidence evaluation are provided in Additional file 1.

\section{Characteristics of apps and websites}

Ten of the 13 apps were commercially developed and three were developed by universities. Six were available on both Android and iOS platforms, four were iOS only, and three were Android only. Of the 12 apps currently 


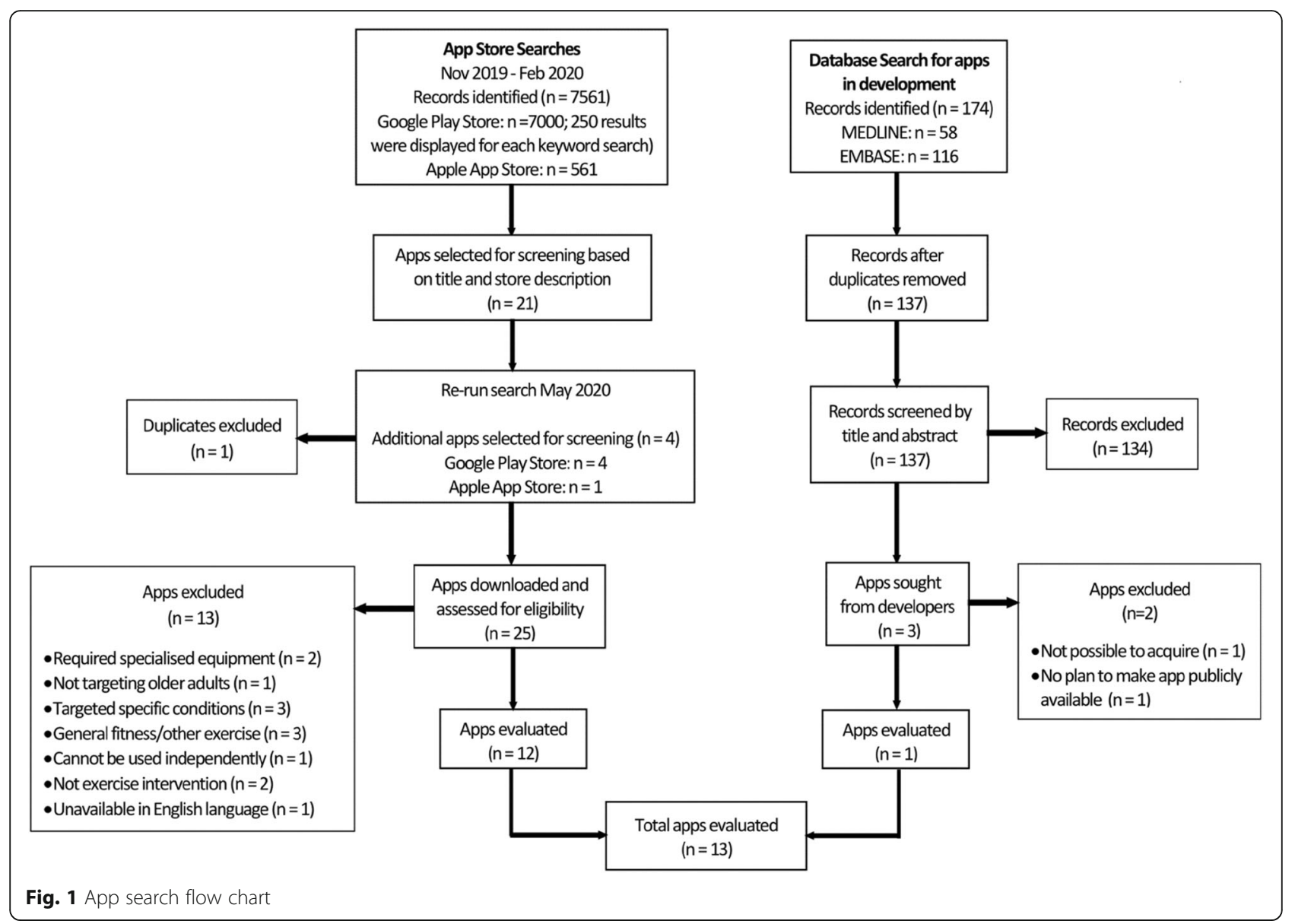

available for download, eight were free to use, and four involved a subscription service. Most of the 24 websites originated from the USA $(n=11)$ or the UK $(n=6)$, with the remainder from Canada $(n=4)$, Australia $(n=1)$, Singapore $(n=1)$, and Europe $(n=1)$. Thirteen were provided by commercial organisations, six were provided by government, three were not-for profit, and two were academic. Detailed characteristics are provided in Additional file 1.

\section{App evaluation}

Table 1 provides an evaluation summary of the 13 apps. On average, total MARS scores differed between raters by 0.57 points $(\mathrm{SD}=0.38$; difference range $=0.02-1.30$ ). Inter-rater agreement between raters on $\mathrm{BCT}$ scores was strong (Kalpha $=0.89 ; 95 \%$ CI: 0.78, 0.97).

\section{Use of evidence-based exercises}

None of the apps have direct evidence supporting their effectiveness, and only one has recently been assessed using an RCT design, with results yet to be published (StandingTall). In seven apps, most of the promoted exercises featured in evidence-based exercise programmes, such as Otago and FaME (see Additional file 1 for information on the types of exercises included in each app). Five apps included at least some evidence-based exercises. One app (Senior Beginner Workout) did not contain any evidence-based exercises. Only one of the apps was explicitly based on an existing evidence-based exercise programme: the Otago Exercise Programme app (Otago).

\section{Use of BCTs}

The mean number of individual BCTs across all apps was five ( $\mathrm{SD}=2.30$; range: $1.5-8.5$ ). Figure 3 (panel a) illustrates the prevalence of BCTs across apps. Frequently included BCTs belonged to the following categories of the 93-item BCT taxonomy: shaping knowledge (12 out of 13 apps); and comparison of behaviour (11 out of 13 apps). Video-based apps (Wysefit and Spiro100) contained the fewest BCTs. Apps containing more than five individual BCTs (the top $50 \%$ in terms of the number of BCTs applied) included StandingTall, Moves4Me, LifeCurve, Otago Exercise Programme, Nymbl Balance, and Keep On Keep Up.

\section{MARS quality ratings}

The mean total MARS score was 3.56 out of $5(\mathrm{SD}=0.32)$. Scores ranged from 2.78 (StopFalls) to 4.09 (Nymbl 
Website Search

November 2019

Screened first 20 results

returned by each of the 28

searches using Google Search

Engine

$(n=560)$
Website Search

May 2020

Screened first 20 results

returned by each of the 28

searches using Google Search

Engine

$(n=560)$

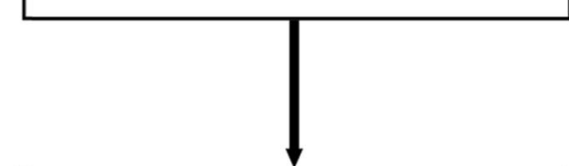

Websites retained for analysis, following exclusion of duplicates and irrelevant sites

( $n=12$ )
Websites retained for analysis,

following exclusion of duplicates and irrelevant sites $(n=20)$

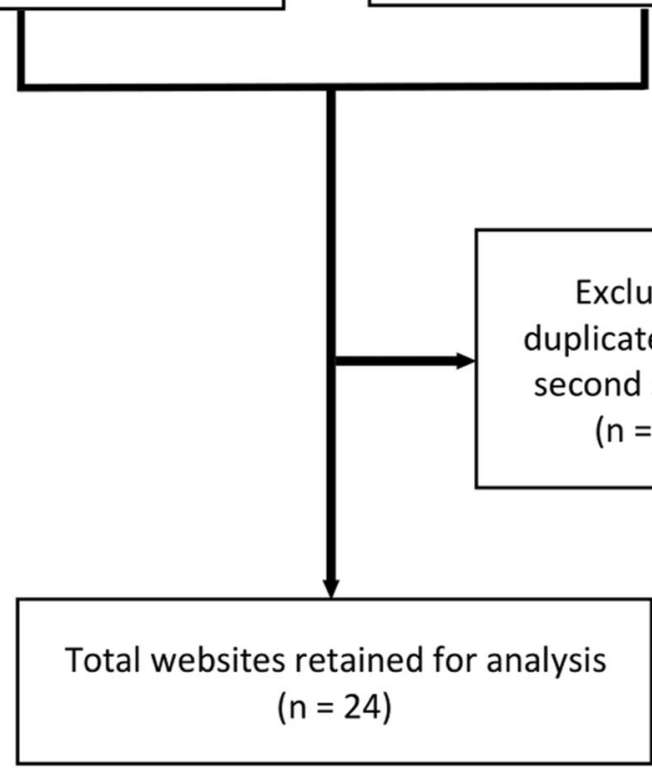

Fig. 2 Website search flow chart

Balance). Most of the apps (8 out of 13) received a score of 'good', but two of these (LifeCurve and Stannah Balance) could be interpreted as 'acceptable-good' with scores falling almost exactly in between the two categories. Five of the apps were rated as 'acceptable' (Table 1).

\section{Website evaluation}

Table 1 provides an evaluation summary for the 24 websites. Kalpha agreement was acceptable for credibility (0.77; 95\% CI: 0.60, 0.89), and strong for senior friendliness $(0.88 ; 95 \% \mathrm{CI}: 0.83,0.92)$ and $\mathrm{BCT}$ ratings (0.85; 95\% CI: 0.73-0.95).

\section{Use of evidence-based exercises}

As expected, none of the websites have been subject to randomised trial design, or other evaluations of effectiveness. In 21 of the websites, most of the featured exercises also appear in evidence-based programmes. Two of the websites included at least some evidence-based exercises. Only one website (closingthegap.ca) did not appear to contain any evidence-based exercises. Three of the 
Table 1 App and website evaluation summary

\begin{tabular}{|c|c|c|c|c|}
\hline App/website name & $\begin{array}{l}\text { Evidence for exercise } \\
\text { intervention } \\
\text { Yes/no/unclear }^{\mathrm{a}}\end{array}$ & $\begin{array}{l}\text { Evidence of effectiveness } \\
\text { of app/website } \\
\text { Yes/no }\end{array}$ & $\begin{array}{l}\text { Mean BCT Score (SD) } \\
\text { Score out of } 93 \text { items }\end{array}$ & $\begin{array}{l}\text { Quality Rating (MARS score } \\
\text { or website quality score) }\end{array}$ \\
\hline \multicolumn{5}{|l|}{ Apps } \\
\hline Exercise Plan for Seniors & Unclear & No & $2.00(0.00)$ & Good (3.60) \\
\hline Hearty Seniors & Unclear & No & $4.00(0.00)$ & Acceptable (3.37) \\
\hline Keep On Keep Up & Yes & No & $6.50(0.50)$ & Good (4.02) \\
\hline LifeCurve & Yes & No & $7.50(0.50)$ & Acceptable/Good (3.50) \\
\hline Moves4Me & Unclear & No & $8.00(0.00)$ & Acceptable (3.46) \\
\hline Nymbl Balance ${ }^{b}$ & Yes & No & $6.50(0.50)$ & Good (4.09) \\
\hline Otago Exercise Programme & Yes & No & $6.50(0.50)$ & Good (3.57) \\
\hline Senior Beginner Workout & No & No & $4.00(0.00)$ & Acceptable (3.45) \\
\hline Spiro100 & Unclear & No & $2.00(0.00)$ & Acceptable (3.38) \\
\hline Standing Tall ${ }^{\mathrm{C}}$ & Yes & No & $8.50(0.50)$ & Good (3.78) \\
\hline Stannah Balance & Yes & No & $4.50(0.50)$ & Acceptable/Good (3.50) \\
\hline StopFalls & Yes & No & $3.50(0.50)$ & Acceptable (2.78) \\
\hline Wysefit & Unclear & No & $1.50(1.50)$ & Good (3.83) \\
\hline \multicolumn{5}{|l|}{ Websites } \\
\hline ageuk.org.uk & Yes & No & $3.00(0.00)$ & Excellent (87\%) \\
\hline betterhealthwhileaging.net & Yes & No & $4.00(0.00)$ & Good (66\%) \\
\hline buffalorehab.com & Yes & No & $4.50(0.50)$ & Good (68\%) \\
\hline caregiverstress.com & Yes & No & $3.00(0.00)$ & Good (72\%) \\
\hline caringseniorservice.com & Yes & No & $2.50(0.50)$ & Fair (50\%) \\
\hline closingthegap.ca & No & No & $2.00(0.00)$ & Fair (58\%) \\
\hline csp.org.uk & Yes & No & $5.00(0.00)$ & Excellent (86\%) \\
\hline dailycaring.com & Yes & No & $4.00(0.00)$ & Good (71\%) \\
\hline eldergym.com & Yes & No & $5.00(0.00)$ & Fair (60\%) \\
\hline exerciseright.com.au & Yes & No & $2.00(0.00)$ & Excellent (81\%) \\
\hline fallsassistant.org.uk & Yes & No & $7.00(0.00)$ & Excellent (79\%) \\
\hline go4life.nia.nih.gov & Yes & No & $7.00(0.00)$ & Good (75\%) \\
\hline healthhub.sg & Yes & No & $4.50(0.50)$ & Good (73\%) \\
\hline healthline.com & Yes & No & $4.00(0.00)$ & Excellent (78\%) \\
\hline healthlinkbc.ca & Yes & No & $4.00(0.00)$ & Good (73\%) \\
\hline hopkinsmedicine.org & Yes & No & $2.50(0.50)$ & Good (68\%) \\
\hline mayoclinic.org & Yes & No & $4.00(0.00)$ & Excellent (85\%) \\
\hline melioguide.com & Yes & No & $2.00(1.00)$ & Good (64\%) \\
\hline nhs.uk/live-well & Yes & No & $6.00(0.00)$ & Excellent (81\%) \\
\hline nhsinform.scot & Yes & No & $3.00(1.00)$ & Excellent (83\%) \\
\hline pathsforall.org.uk & Yes & No & $4.00(0.00)$ & Good (72\%) \\
\hline preventfalls.ca & Yes & No & $2.00(1.00)$ & Good (69\%) \\
\hline profound.eu.com & Yes & No & $5.00(0.00)$ & Good (64\%) \\
\hline unitypoint.org & Yes & No & $3.00(0.00)$ & Fair (59\%) \\
\hline
\end{tabular}

Overall quality for apps was assessed using MARS. Interpretation of MARS ratings is based on mean scores rounded to the nearest whole number $(1=$ Inadequate; 2 = Poor; 3 = Acceptable; $4=$ Good; 5 = Excellent). Overall quality for websites was calculated as the mean of the total scores on credibility and senior friendliness and expressed as a percentage (poor = less than $50 \%$ score; fair $=50-62 \%$; good $=63-75 \%$; excellent $=$ greater than $75 \%$ ). BCT, MARS and website quality ratings reflect mean scores of two independent raters

${ }^{\text {a }}$ 'Yes' = Promoted exercises are predominately evidence-based (e.g. feature in FaME or Otago programmes); 'No' = Few/none of the exercises feature in evidence-based programmes; 'unclear' = some of the exercises feature in evidence-based programmes

${ }^{b}$ Only available for download in USA

${ }^{\mathrm{C}}$ In development and not yet publicly available 


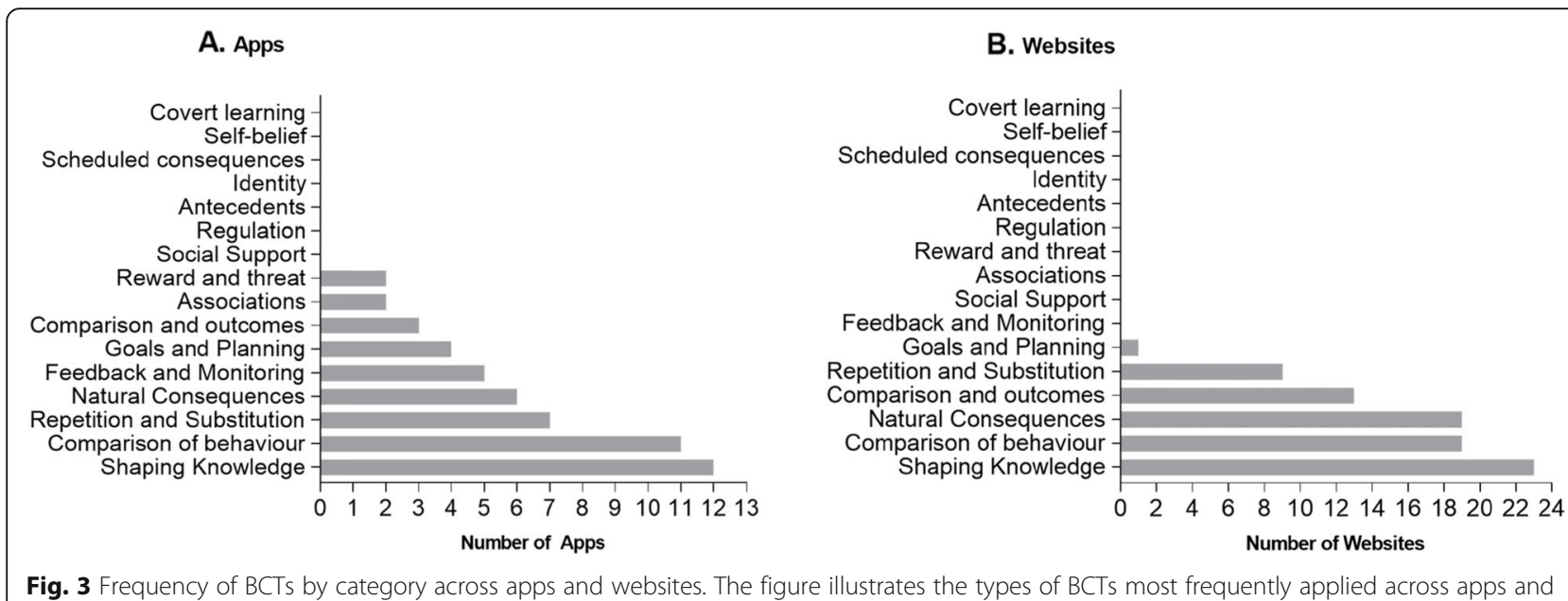

Fig. 3 Frequency of BCTs by category across apps and websites. The figure illustrates the types of BCTs most frequently applied across apps and websites, based on categories provided in the 93-item BCT Taxonomy

websites (profound.eu.com, betterhealthwhileaging.net, and caringseniorservice.com) contained video demonstrations of the Otago Exercise Programme, for which there is a strong evidence-base for effectiveness (see Additional file 1 for information on the types of exercises demonstrated by each website).

\section{Use of BCTs}

The mean number of individual BCTs across all websites was 3.88 ( $\mathrm{SD}=1.44$; range: $2-7$ ). Figure 3 (panel b) illustrates the prevalence of $\mathrm{BCTs}$ across websites. Frequently included BCTs belonged to the following categories of the 93-item BCT taxonomy: shaping knowledge (23 out of 24 websites); comparison of behaviour (19 out of 24); and natural consequences (19 out of 24). Websites containing more than 4.5 individual BCTs (the top $50 \%$ in terms of number of BCTs applied) included buffalorehab.com, csp.org.uk, eldergym.com, fallsassistant.org.uk, go4life.nia.nih.gov, healthhub.sg, nhs.uk/live-well, and profound.eu.com.

\section{Quality of websites}

Overall, the websites scored an average of $71.74 \%$, indicating good quality. Eight were considered excellent, 12 were considered good, four were considered fair, and none of the websites were considered poor. The mean credibility score across all websites was 4.60 out of a possible $7(65.77 \%)(\mathrm{SD}=1.15$; range $=2-7)$, indicating that compliance with the HONCode standards for health-related websites was good. Only two websites stated that they were HONCode compliant, with mayoclinic.org meeting all seven standards, and healthline.com meeting six. Three of the websites (caringseniorservice.com, eldergym.com, and unitypoint.org), scored poorly on credibility (score $<50 \%$ ) The mean senior friendliness score across all websites was 31.08 out of a possible 40
$(77.71 \%) \quad(\mathrm{SD}=4.13 ; \quad$ range $=23-38)$, indicating that overall website senior friendliness was excellent. None of the websites scored lower than $20(<50 \%)$ on senior friendliness.

\section{Discussion}

There is robust evidence for the effectiveness of community-based strength and balance exercise programmes in falls prevention, provided such programmes meet the minimum requirement of 50 contact hours over a 24 week period [5]. However, these types of structured programmes might not be feasible or accessible to many older adults living in the community, particularly during the COVID-19 pandemic where many older people are practising physical distancing. Digital approaches, such as apps and websites, offer a means of facilitating strength and balance exercises independently in the home. This review provides an overview and evidence summary of apps and websites that are publicly available or currently in development, to support older adult engagement in strength and balance exercises. We determined which of these apps appear most appropriate for home-based strength and balance exercise by identifying which apps scored well across our key evaluation criteria (Table 1). Of the seven apps that contained exercises with an evidence base in falls prevention, four also received a "good" MARS quality rating. These four apps (StandingTall, Otago Exercise Programme, Nymbl Balance, and Keep On Keep Up) also scored comparatively well on use of BCTs (i.e. scored in the top $50 \%$ in terms of the number of BCTs applied). Two of these apps are currently available for public download in the UK (Otago Exercise Programme and Keep On Keep Up) and could be recommended for use by older people who wish to engage in strength and balance exercises at home, particularly under the current pandemic conditions. Nymbl 
Balance may also be a useful tool, but it is currently only available for download in the USA. The StandingTall app is still under development and only available to services which have joined the Standing Tall-er Implementation Study (NIHR CPMS ID: 44434; IRAS ID 268954). Of note, none of these apps have published findings in relation to effectiveness in falls prevention, and it's possible that although evidence-based exercises are promoted, online delivery of these programmes may lack key motivational and support elements present in faceto-face delivery.

We determined which websites may be most appropriate for independent strength and balance exercise by identifying which websites scored well across our key evaluation criteria (Table 1). Of the 23 websites that contained exercises with an evidence base in falls prevention, eight also received an "excellent" quality rating. Three of these websites also scored in the top 50\% in terms of number of BCTs applied (csp.org.uk, fallsassistant.org.uk, and nhs.uk/live-well). These websites could be recommended to facilitate older adult engagement in strength and balance exercises in their own homes. Three additional websites (profound.eu.com, betterhealthwhileaging.net, and caringseniorservice.com) contained video demonstrations of the Otago exercise programme which has a strong evidence base. These websites may act as a substitute for face-to-face delivery for older people wishing to undertake evidence-based falls prevention exercise programmes in their own homes. The majority of the websites demonstrated some of the exercises that feature in falls prevention programmes with a strong evidence-base and as such, may prove useful for older adults wishing to engage in strength and balance training at home. However, as none of these websites have been evaluated in the context of an RCT or other study design, their effectiveness in falls prevention remains unknown. A key advantage of web-based videos and images is that they do not require a smartphone or tablet and are freely available to those with access to an internet-connected computer.

A strength of this review is the comprehensive search strategy and detailed quality appraisal of the featured apps and websites. Findings may also provide a useful summary for healthcare professionals looking for alternatives to face-to-face delivery of exercise programmes, particularly in the context of COVID-19. Exercise programmes are usually delivered by trained professionals during face-to-face classes or home visits, but this has largely been curtailed by COVID-19 lock down. This is a concern as the benefits of exercise are known to be lost rapidly once exercise is stopped [33]. Preliminary findings from this review were presented to the Department of Health and Social Care (DHSC) in the UK as part of a policy briefing on the digital delivery of strength and balance exercise training in response to increased home isolation and physical distancing [34]. Our report recommended that the apps and websites found to be of high quality following our evaluation could potentially be used as substitutes for face-to-face delivery. In addition, the recent Public Health England (PHE) prevention green paper [35] proposed to prioritise digital approaches in supporting the public to engage in strength and balance exercises regularly. Their aim is to provide digital products or services that are freely available to everyone, and support older people in particular, as well as those living with health conditions or people on low income. The apps and websites identified in this review could help to achieve PHE's long-term aim, while also supporting older people to remain physically active during the COVID-19 pandemic. All of the recommended resources contain exercises used in existing face-to-face evidence-based programmes, although there is no evidence that these exercises are effective in falls prevention when delivered via a digital platform. Even so, the roll out of digital strength and balance exercises is preferable to older people not receiving any such interventions for the duration of the COVID-19 pandemic. The rigorous approach to app and website evaluation used in this review could be used by public health bodies to identify digital resources and build a "menu" for older people, carers, and healthcare professionals to select high quality, evidence-based digital tools that could facilitate exercising at home.

Even so, digital approaches may only act as a suitable alternative for generally healthy older people with experience using such technologies. Many older people may not have access to the internet, or internet-enabled mobile devices, particularly those from older age cohorts and poorer socio-economic groups, or those with declining visual acuity or cognitive function [36]. Furthermore, independent exercise may not be suitable for everyone, such as older adults with poor general health, frailty, or fear of falling [37]. This review was limited to searching for apps and websites in the UK, and as such, findings may not be generalisable internationally. In addition, as an indicator of quality, we assessed the extent to which apps and websites drew on BCTs using a simple count of the number of BCTs present according to a 93-item taxonomy [21]. Although several BCTs typically occur together in a given intervention and it is likely that the effect of a single BCT will be small, [38], the effect of specific BCT combinations in the context of app- and website-based exercise interventions on fall-related outcomes remains unclear. As such, conclusions about the behaviour change potential of these apps/websites due to BCT utilisation should be interpreted cautiously.

There is a need for a more thorough understanding of the effectiveness of digital approaches in falls prevention 
and this can be gained through RCTs of app- and webbased interventions. Websites may be more difficult to evaluate in a controlled manner as they are engaged with by users to varying degrees and under a range of conditions [39]. Despite these limitations, apps and websites have the potential to provide a convenient, costeffective, and accessible means for many older adults to engage in strength and balance training independently and reduce falls risk.

\section{Conclusions}

Apps and websites offer a means of facilitating strength and balance exercises independently in the home and may prove particularly useful as alternatives to face-toface delivery of exercise programmes in the context of COVID-19. There are a number of high quality apps and websites currently available for use by older people who wish to engage in falls prevention exercises. Nevertheless, RCT evaluations of these kinds of approaches in falls prevention are needed.

\section{Supplementary Information}

Supplementary information accompanies this paper at https://doi.org/10 1186/s12877-020-01880-6.

Additional file 1. Additional Information.

\section{Abbreviations}

BCTs: Behaviour change techniques; HONCode: Health on the Net Code of Conduct for Medical Health Websites; MARS: Mobile Application Rating Scale; PHE: Public Health England; PRISMA: Preferred Reporting Items for Systematic Reviews and Meta-Analysis; RCT: Randomised controlled trial

\section{Acknowledgements}

The authors thank Julia Mueller, Rebecca Symes, Karen Luker, David Hegarty, and Monserrat Conde for assisting with MARS ratings for the apps included in this review.

\section{Authors' contributions}

All authors were involved in the conception and design of the review. LM performed the app, website, and literature search and data analyis. All authors were involved in screening, data extraction and interpretation. LM drafted the initial manuscript and $E B$ and $C T$ provided critical revisions. All authors read and approved the final manuscript.

Authors' information

Not applicable.

\section{Funding}

This work was supported by the National Institute for Health Research through the NIHR Policy Research Unit in Older People and Frailty, NIHR Applied Research Collaboration Greater Manchester, and a NIHR Senior Investigator Award (Reference NIHR200299) to Prof Todd. The views expressed are those of the authors and not necessarily those of the NHS, the NIHR, the Department of Health and Social Care, or its partner organisations.

\section{Availability of data and materials}

Not applicable.

Ethics approval and consent to participate Not applicable.
Consent for publication

Not applicable.

\section{Competing interests}

Prof Todd declares that he is PI for the UK arm of the Standing Taller implementation evaluation funded by the Australian National Health and Medical Research Council, was the PI for the EC funded Prevention of Falls Network for Dissemination (ProFouND), which developed and maintained the www.profound.eu.com website. Prof Todd and Elisabeth Boulton were Cls on the EC funded PreventIT project that created the eLiFE app. The Keep On Keep Up app is being developed by researchers at the University of Manchester along with Reason Digital. None of the authors have any financial stake in any of the apps or websites reviewed. Lisa McGarrigle declares no conflict of interest.

\section{Author details}

${ }^{1}$ School of Health Sciences, Faculty of Biology, Medicine and Health, The University of Manchester, Jean McFarlane Building, University Place, Oxford Road, Manchester M13 9PL, UK. ${ }^{2}$ Manchester Academic Health Science Centre, Manchester M13 9NQ, UK. ${ }^{3}$ National Institute for Health Research (NIHR) Older People and Frailty Policy Research Unit, School of Health Sciences, Faculty of Biology, Medicine and Health, The University of Manchester, Manchester M13 9PL, UK. ${ }^{4}$ Manchester University NHS Foundation Trust, Manchester M13 9WL, UK. ${ }^{5}$ National Institute for Health Research, Applied Research Collaboration- Greater Manchester, Manchester M13 9PL, UK.

Received: 11 August 2020 Accepted: 9 November 2020

Published online: 18 November 2020

\section{References}

1. World Health Organization. Falls: key facts. 2018. https://www.who.int/newsroom/fact-sheets/detail/falls. Accessed 17 Mar 2020.

2. Frieson CW, Tan MP, Ory MG, Smith ML. Editorial: evidence-based practices to reduce falls and fall-related injuries among older adults. Front Public Health. 2018;6:222

3. Gillespie LD, Robertson MC, Gillespie WJ, Sherrington C, Gates S, Clemson $L M$, et al. Interventions for preventing falls in older people living in the community. Cochrane Database Syst Rev. 2012;(9):CD007146. https://doi. org/10.1002/14651858.CD007146.pub3.

4. Kinney JM. Nutritional frailty, sarcopenia and falls in the elderly. Curr Opin Clin Nutr Metab Care. 2004;7:15.

5. Sherrington C, Fairhall NJ, Wallbank GK, Tiedemann A, Michaleff ZA, Howard $\mathrm{K}$, et al. Exercise for preventing falls in older people living in the community. Cochrane Database Syst Rev. 2019. https://doi.org/10.1002/ 14651858.CD012424.pub2.

6. Department of Health and Social Care. UK chief medical officers' physical activity guideline. 2019. https://www.gov.uk/government/publications/ physical-activity-guidelines-uk-chief-medical-officers-report. Accessed $30 \mathrm{Mar}$ 2020.

7. Office for National Statistics. Health survey for England 2016. London: Office for National Statistics; 2017. https://digital.nhs.uk/data-and-information/ publications/statistical/health-survey-for-england/health-survey-forengland-2016. Accessed 7 June 2020.

8. Muellmann S, Forberger S, Mollers T, Broring E, Zeeb H, Pischke CR. Effectiveness of eHealth interventions for the promotion of physical activity in older adults: a systematic review. Prev Med. 2018;108:93-110.

9. Yerrakalva D, Yerrakalva D, Hajna S, Griffin S. Effects of mobile health app interventions on sedentary time, physical activity, and fitness in older adults: systematic review and meta-analysis. J Med Internet Res. 2019;21. https:// doi.org/10.2196/14343.

10. Sato K, Kuroki K, Saiki S, Nagatomi R. Improving walking, muscle strength, and balance in the elderly with an Exergame using Kinect: a randomized controlled trial. Games Health J. 2015:4:161-7.

11. Stanmore EK, Mavroeidi A, de Jong LD, Skelton DA, Sutton CJ, Benedetto V, et al. The effectiveness and cost-effectiveness of strength and balance Exergames to reduce falls risk for people aged 55 years and older in UK assisted living facilities: a multi-centre, cluster randomised controlled trial. BMC Med. 2019;17:49.

12. Lamb SE, Becker C, Gillespie LD, Smith JL, Finnegan S, Potter R, et al. Reporting of complex interventions in clinical trials: development of a 
taxonomy to classify and describe fall-prevention interventions. Trials. 2011; 12:125.

13. Gardner MM, Buchner DM, Robertson MC, Campbell AJ. Practical implementation of an exercise-based falls prevention programme. Age Ageing. 2001;30:77-83.

14. Featherstone RM, Dryden DM, Foisy M, Guise J-M, Mitchell MD, Paynter RA et al. Advancing knowledge of rapid reviews: an analysis of results, conclusions and recommendations from published review articles examining rapid reviews. Syst Rev. 2015;4:50.

15. Khangura S, Konnyu K, Cushman R, Grimshaw J, Moher D. Evidence summaries: the evolution of a rapid review approach. Syst Rev. 2012;1:10.

16. Eysenbach $G$, Köhler C. How do consumers search for and appraise health information on the world wide web? Qualitative study using focus groups, usability tests, and in-depth interviews. BMJ. 2002;324:573-7.

17. Spink A, Jansen BJ, Wolfram D, Saracevic T. From e-sex to e-commerce: web search changes. Computer. 2002;35:107-9

18. Whitehead SH, Nyman SR, Broaders F, Skelton DA, Todd CJ. The quality of English-language websites offering falls-prevention advice to older members of the public and their families. Health Informatics J. 2012;18:50-65.

19. Skelton D, Dinan S, Campbell M, Rutherford O. Tailored group exercise (Falls Management Exercise -- FaME) reduces falls in community-dwelling older frequent fallers (an RCT). Age Ageing. 2005;34:636-9.

20. Campbell AJ, Robertson MC. Otago exercise programme to prevent falls in older adults. Wellington: ACC Thinksafe; 2003. p. 3.

21. Michie S, Richardson M, Johnston M, Abraham C, Francis J, Hardeman W, et al. The behavior change technique taxonomy (v1) of 93 hierarchically clustered techniques: building an international consensus for the reporting of behavior change interventions. Ann Behav Med Publ Soc Behav Med. 2013:46:81-95.

22. Stoyanov SR, Hides L, Kavanagh DJ, Zelenko O, Tjondronegoro D, Mani M Mobile app rating scale: a new tool for assessing the quality of health mobile apps. JMIR MHealth UHealth. 2015:3:e27.

23. Higgins JPT, Altman DG, Gøtzsche PC, Jüni P, Moher D, Oxman AD, et al. The Cochrane Collaboration's tool for assessing risk of bias in randomised trials. BMJ. 2011;343. https://doi.org/10.1136/bmj.d5928.

24. Sterne JA, Hernán MA, Reeves BC, Savović J, Berkman ND, Viswanathan M, et al. ROBINS-l: a tool for assessing risk of bias in non-randomised studies of interventions. BMJ. 2016:355. https://doi.org/10.1136/bmj.i4919.

25. Health on the Net Foundation. HON Code of Conduct (HONCode) for medical and health websites. 2019. https://www.hon.ch/cgi-bin/HONcode/ principles.pl?English. Accessed 7 Apr 2020.

26. Hayes AF, Krippendorff K. Answering the call for a standard reliability measure for coding data. Commun Methods Meas. 2007;1:77-89.

27. De Swert K. Calculating inter-coder reliability in media content analysis using Krippendorff's Alpha. The Netherlands: University of Amsterdam; 2012. http://www.polcomm.org/wp-content/uploads/ICR01022012.pdf. Accessed 17 July 2020.

28. Moore GF, Audrey S, Barker M, Bond L, Bonell C, Hardeman W, et al. Process evaluation of complex interventions: Medical Research Council guidance. BMJ. 2015;350. https://doi.org/10.1136/bmj.h1258.

29. van Het Reve E, Silveira P, Daniel F, Casati F, de Bruin ED. Tablet-based strength-balance training to motivate and improve adherence to exercise in independently living older people: part 2 of a phase II preclinical exploratory trial. J Med Internet Res. 2014;16:e159.

30. Delbaere K, Valenzuela T, Woodbury A, Davies T, Yeong J, Steffens D, et al. Evaluating the effectiveness of a home-based exercise programme delivered through a tablet computer for preventing falls in older community-dwelling people over 2 years: study protocol for the standing tall randomised controlled trial. BMJ Open. 2015;5. https://doi.org/10.1136/ bmjopen-2015-009173.

31. Taraldsen K, Mikolaizak AS, Maier AB, Boulton E, Aminian K, van Ancum J, et al. Protocol for the PreventIT feasibility randomised controlled trial of a lifestyle-integrated exercise intervention in young older adults. BMJ Open. 2019:9:e023526.

32. Taraldsen K, Mikolaizak AS, Maier AB, Mellone S, Boulton E, Aminian K, et al. Digital technology to deliver a lifestyle-integrated exercise intervention in young seniors - the PreventIT feasibility randomised controlled trial. Front Digit Health. 2020;2. https://doi.org/10.3389/fdgth.2020.00010.

33. Sherrington C, Tiedemann A, Fairhall N, Close JCT, Lord SR. Exercise to prevent falls in older adults: an updated meta-analysis and best practice recommendations. New South Wales Public Health Bull. 2011;22:78-83.
34. NIHR Policy Research Unit Older People and Frailty. Delivery of strength and balance exercises for falls prevention amongst older people using digital technologies to replace face-to-face contact during Covid-19 home isolation and physical distancing: briefing paper. 2020. https://www.opfpru. nihr.ac.uk/covid-19-research/rr7-covid-19-technology-for-strength-andbalance/. Accessed 7 Oct 2020.

35. Public Health England. Advancing our health: prevention in the 2020s. London: Public Health England; 2019. https://www.gov.uk/government/ consultations/advancing-our-health-prevention-in-the-2020s. Accessed 7 June 2020.

36. Matthews K, Nazroo J, Marshall A. Digital inclusion in later life: cohort changes in internet use over a ten-year period in England. Ageing Soc. 2019:39:1914-32.

37. Franco MR, Tong A, Howard K, Sherrington C, Ferreira PH, Pinto RZ, et al. Older people's perspectives on participation in physical activity: a systematic review and thematic synthesis of qualitative literature. Br J Sports Med. 2015;49:1268-76.

38. Michie S, West R, Sheals K, Godinho CA. Evaluating the effectiveness of behavior change techniques in health-related behavior: a scoping review of methods used. Transl Behav Med. 2018;8:212-24.

39. Brusse C, Gardner K, McAullay D, Dowden M. Social media and mobile apps for health promotion in Australian indigenous populations: scoping review. J Med Internet Res. 2014;16:e280

\section{Publisher's Note}

Springer Nature remains neutral with regard to jurisdictional claims in published maps and institutional affiliations.
Ready to submit your research? Choose BMC and benefit from:

- fast, convenient online submission

- thorough peer review by experienced researchers in your field

- rapid publication on acceptance

- support for research data, including large and complex data types

- gold Open Access which fosters wider collaboration and increased citations

- maximum visibility for your research: over $100 \mathrm{M}$ website views per year

At $\mathrm{BMC}$, research is always in progress.

Learn more biomedcentral.com/submissions 